International Journal of Pure and Applied Mathematics

Volume 103 No. 4 2015, 709-718

ISSN: 1311-8080 (printed version); ISSN: 1314-3395 (on-line version)

url: http://www.ijpam.eu

doi: http://dx.doi.org/10.12732/ijpam.v103i4.10

\title{
COMMON FIXED POINTS FOR INTIMATE MAPPINGS IN MULTIPLICATIVE METRIC SPACES
}

\author{
Parveen Kumar ${ }^{1}$, Sanjay Kumar², Shin Min Kang ${ }^{3}$ \\ ${ }^{1,2}$ Department of Mathematics \\ Deenbandhu Chhotu Ram University of Science and Technology \\ Murthal, Sonipat 131039, Haryana, INDIA \\ ${ }^{3}$ Department of Mathematics and RINS \\ Gyeongsang National University \\ Jinju, 660-701, KOREA
}

\begin{abstract}
In this paper, we introduce the notion of intimate mappings in multiplicative metric spaces and prove common fixed point theorem for these mappings.
\end{abstract}

AMS Subject Classification: 47H10, 54H25

Key Words: multiplicative metric spaces, compatible mappings of type $(A)$, intimate mappings

\section{Introduction and Preliminaries}

The letters $\mathbb{R}, \mathbb{R}_{+}$and $\mathbb{N}$ denote the set of all real numbers, the set of all positive real numbers and the set of all natural numbers, respectively.

In 2008, Bashirov et al. [2] introduced the concept of multiplicative metric spaces as follows:

Definition 1.1. Let $X$ be a nonempty set. A multiplicative metric is a mapping $d: X \times X \rightarrow \mathbb{R}_{+}$satisfying the following conditions:

(i) $d(x, y) \geq 1$ for all $x, y \in X$ and $d(x, y)=1$ if and only if $x=y$;

Received: June 23, 2015

$\S_{\text {Correspondence author }}$ (c) 2015 Academic Publications, Ltd. url: www.acadpubl.eu 
(ii) $d(x, y)=d(y, x)$ for all $x, y \in X$;

(iii) $d(x, y) \leq d(x, z) \cdot d(z, y)$ for all $x, y, z \in X$ (multiplicative triangle inequality).

Then the mapping $d$ together with $X$, that is, $(X, d)$ is a multiplicative metric space.

Example 1.2. ([6]) Let $\mathbb{R}_{+}^{n}$ be the collection of all $n$-tuples of positive real numbers. Let $d^{*}: \mathbb{R}_{+}^{n} \times \mathbb{R}_{+}^{n} \rightarrow \mathbb{R}$ be defined as follows:

$$
d^{*}(x, y)=\left|\frac{x_{1}}{y_{1}}\right|^{*} \cdot\left|\frac{x_{2}}{y_{2}}\right|^{*} \cdots\left|\frac{x_{n}}{y_{n}}\right|^{*},
$$

where $x=\left(x_{1}, \ldots, x_{n}\right), y=\left(y_{1}, \ldots, y_{n}\right) \in \mathbb{R}_{+}^{n}$ and $|\cdot|^{*}: \mathbb{R}_{+} \rightarrow \mathbb{R}_{+}$is defined by

$$
|a|^{*}= \begin{cases}a & \text { if } a \geq 1 \\ \frac{1}{a} & \text { if } a<1 .\end{cases}
$$

Then it is obvious that all conditions of a multiplicative metric are satisfied. and $\left(\mathbb{R}_{+}^{n}, d\right)$ is a multiplicative metric space.

Example 1.3. ([8]) Let $d: \mathbb{R} \times \mathbb{R} \rightarrow[1, \infty)$ be defined as $d(x, y)=a^{|x-y|}$, where $x, y \in \mathbb{R}$ and $a>1$. Then $d$ is a multiplicative metric and $(\mathbb{R}, d)$ is a multiplicative metric space.

Remark 1.4. We note that the Example 1.2 is valid for positive real numbers and Example 1.3 is valid for all real numbers.

Example 1.5. ([8]) Let $(X, d)$ be a metric space. Define a mapping $d_{a}$ on $X$ by

$$
d_{a}(x, y)=a^{d(x, y)}= \begin{cases}1 & \text { if } x=y, \\ a & \text { if } x \neq y,\end{cases}
$$

where $x, y \in X$ and $a>1$. Then $d_{a}$ is called a discrete multiplicative metric and $\left(X, d_{a}\right)$ is known as the discrete multiplicative metric space.

Example 1.6. ([1]) Let $X=C^{*}[a, b]$ be the collection of all real-valued multiplicative continuous functions on $[a, b] \subset \mathbb{R}_{+}$. Then $(X, d)$ is a multiplicative metric space with $d$ defined by $d(f, g)=\sup _{x \in[a, b]}\left|\frac{f(x)}{g(x)}\right|$ for arbitrary $f, g \in X$. 
Remark 1.7. ([8]) Neither every metric is multiplicative metric nor every multiplicative metric is metric. The mapping $d^{*}$ defined above is multiplicative metric but not metric as it does not satisfy triangular inequality. Consider

$$
d^{*}\left(\frac{1}{3}, \frac{1}{2}\right)+d^{*}\left(\frac{1}{2}, 3\right)=\frac{3}{2}+6=7.5<9=d^{*}\left(\frac{1}{3}, 3\right) .
$$

On the other hand the usual metric on $\mathbb{R}$ is not multiplicative metric as it doesnt satisfy multiplicative triangular inequality, since

$$
d(2,3) \cdot d(3,6)=3<4=d(2,6) .
$$

One can refer to $[4,6]$ for detailed multiplicative metric topology.

Definition 1.8. Let $(X, d)$ be a multiplicative metric space. Then a sequence $\left\{x_{n}\right\}$ in $X$ said to be

(1) a multiplicative convergent to $x$ if for every multiplicative open ball $B_{\epsilon}(x)=\{y \in X \mid d(x, y)<\epsilon\}, \epsilon>1$, there exists a natural number $N$ such that $n \geq N$, then $x_{n} \in B_{\epsilon}(x)$, that is, $d\left(x_{n}, x\right) \rightarrow 1$ as $n \rightarrow \infty$.

(2) a multiplicative Cauchy sequence if for all $\epsilon>1$, there exists $N \in \mathbb{N}$ such that $d\left(x_{n}, x_{m}\right)<\epsilon$ for all $m, n>N$, that is, $d\left(x_{n}, x_{m}\right) \rightarrow 1$ as $n, m \rightarrow \infty$.

A multiplicative metric space is said to be complete if every multiplicative Cauchy sequence in it is multiplicative convergent to $x \in X$.

Remark 1.9. The set of positive real numbers $\mathbb{R}_{+}$is not complete according to the usual metric. Let $X=\mathbb{R}_{+}$and the sequence $\left\{x_{n}\right\}=\left\{\frac{1}{n}\right\}$. It is obvious $\left\{x_{n}\right\}$ is a Cauchy sequence in $X$ with respect to usual metric and $X$ is not a complete metric space, since $0 \notin \mathbb{R}_{+}$. In case of a multiplicative metric space, we take a sequence $\left\{x_{n}\right\}=\left\{a^{\frac{1}{n}}\right\}$, where $a>1$. Then $\left\{x_{n}\right\}$ is a Cauchy sequence since for $n \geq m$,

$$
\begin{aligned}
d\left(x_{n}, x_{m}\right) & =\left|\frac{x_{n}}{x_{m}}\right|=\left|\frac{a^{\frac{1}{n}}}{a^{\frac{1}{m}}}\right|=\left|a^{\frac{1}{n}-\frac{1}{m}}\right| \\
& \leq a^{\frac{1}{m}-\frac{1}{n}}<a^{\frac{1}{m}}<\epsilon \quad \text { if } m>\frac{\log a}{\log \epsilon},
\end{aligned}
$$

where $|a|=\left\{\begin{array}{ll}a & \text { if } a \geq 1, \\ \frac{1}{a} & \text { if } a<1 .\end{array}\right.$ Also, $\left\{x_{n}\right\} \rightarrow 1$ as $n \rightarrow \infty$ and $1 \in \mathbb{R}_{+}$. Hence $(X, d)$ is a complete multiplicative metric space. 
In 2012, Özavsar and Çevikel [6] gave the concept of multiplicative contraction mappings and proved some fixed point theorem of such mappings in a multiplicative metric space.

Definition 1.10. Let $f$ be a mapping of a multiplicative metric space $(X, d)$ into itself. Then $f$ is said to be a multiplicative contraction if there exists a real constant $\lambda \in[0,1)$ such that

$$
d(f x, f y) \leq d^{\lambda}(x, y) \quad \text { for all } x, y \in X .
$$

$\mathrm{Gu}$ et al. [3] introduced the notion of commutative and weak commutative mappings in a multiplicative metric space and proved some fixed point theorems for these mappings.

Definition 1.11. Let $f$ and $g$ be two mappings of a multiplicative metric space $(X, d)$ into itself. Then $f$ and $g$ are said to be

(1) commutative mappings if $f g x=g f x$ for all $x \in X$.

(2) weak commutative mappings if $d(f g x, g f x) \leq d(f x, g x)$ for all $x \in X$.

Notice that commuting mappings are obviously weakly commuting. However, the converse need not be true.

Example 1.12. Let $X=[0,1]$ be a multiplicative metric $d$ on $X$ defined by $d(x, y)=a^{|x-y|}$, where for all $x, y \in X$ and $a>1$. Define mappings $f$ and $g: X \rightarrow X$ by $f x=\frac{x}{3-x}$ and $g x=\frac{x}{3}$ for all $x \in X$. For any $x \in X$,

$$
d(f g x, g f x)=a^{\left|\frac{2 x^{2}}{(9-x)(9-3 x)}\right|} \leq a^{\left|\frac{x^{2}}{9-3 x}\right|}=d(f x, g x) .
$$

Then $f$ and $g$ are weakly commuting but $f$ and $g$ are not commuting since

$$
f g x=\frac{x}{9-x}<\frac{x}{9-3 x}=g f x
$$

for any non-zero $x \in X$.

\section{Main Results}

Recently, Kang et al. [5] introduced the notion of compatible mappings and its variant in multiplicative metric spaces as follows: 
Definition 2.1. Let $f$ and $g$ be two mappings of a multiplicative metric space $(X, d)$ into itself. Then $f$ and $g$ are said to be

(1) compatible if

$$
\lim _{n \rightarrow \infty} d\left(f g x_{n}, g f x_{n}\right)=1,
$$

whenever $\left\{x_{n}\right\}$ is a sequence in $X$ such that $\lim _{n \rightarrow \infty} f x_{n}=\lim _{n \rightarrow \infty} g x_{n}=t$ for some $t \in X$.

(2) compatible of type $(A)$ if

$$
\lim _{n \rightarrow \infty} d\left(f g x_{n}, g g x_{n}\right)=1 \text { and } \lim _{n \rightarrow \infty} d\left(g f x_{n}, f f x_{n}\right)=1,
$$

whenever $\left\{x_{n}\right\}$ is a sequence in $X$ such that $\lim _{n \rightarrow \infty} f x_{n}=\lim _{n \rightarrow \infty} g x_{n}=t$ for some $t \in X$.

In 2001, Sahu et al. [7] introduced the notion of intimate mappings in metric spaces. In fact it is the generalization of compatible mappings of type (A).

In similar mode, we introduce the notions of intimate mappings in multiplicative metric spaces, which is also a generalization of compatible mappings of type $(A)$ in multiplicative metric spaces.

Definition 2.2. Let $f$ and $g$ be two mappings of a multiplicative metric space $(X, d)$ into itself. Then $f$ and $g$ are said to be

(1) g-intimate mappings if

$$
\alpha d\left(g f x_{n}, g x_{n}\right) \leq \alpha d\left(f f x_{n}, f x_{n}\right),
$$

where $\alpha=\lim$ sup or liminf and $\left\{x_{n}\right\}$ is a sequence in $X$ such that $\lim _{n \rightarrow \infty} f x_{n}=$ $\lim _{n \rightarrow \infty} g x_{n}=t$ for some $t \in X$.

(2) f-intimate mappings if

$$
\alpha d\left(f g x_{n}, f x_{n}\right) \leq \alpha d\left(g g x_{n}, g x_{n}\right),
$$

where $\alpha=\lim \sup$ or $\lim \inf$ and $\left\{x_{n}\right\}$ is a sequence in $X$ such that $\lim _{n \rightarrow \infty} f x_{n}=$ $\lim _{n \rightarrow \infty} g x_{n}=t$ for some $t \in X$.

Proposition 2.3. Let $f$ and $g$ be mappings of a multiplicative metric space $(X, d)$ into itself. Assume that $f$ and $g$ are compatible of type $(A)$. Then $f$ and $g$ are $f$-intimate and $g$-intimate. 
Proof. Since $f$ and $g$ are compatible of type $(A)$, we havw

$$
\lim _{n \rightarrow \infty} d\left(g f x_{n}, f f x_{n}\right)=1 \text { and } \lim _{n \rightarrow \infty} d\left(f g x_{n}, g g x_{n}\right)=1,
$$

whenever $\left\{x_{n}\right\}$ is a sequence in $X$ such that $\lim _{n \rightarrow \infty} f x_{n}=\lim _{n \rightarrow \infty} g x_{n}=t$ for some $t \in X$.

Now

$$
d\left(f g x_{n}, f x_{n}\right) \leq d\left(f g x_{n}, g g x_{n}\right) \cdot d\left(g g x_{n}, f x_{n}\right)
$$

and hence

$$
\begin{aligned}
\alpha d\left(f g x_{n}, f x_{n}\right) & \leq \alpha d\left(g g x_{n}, f g x_{n}\right) \cdot \alpha d\left(g g x_{n}, f x_{n}\right) \\
& =\alpha d\left(g g x_{n}, f g x_{n}\right) \cdot \alpha d\left(g g x_{n}, g x_{n}\right) \\
& =\alpha d\left(g g x_{n}, g x_{n}\right),
\end{aligned}
$$

whenever $\left\{x_{n}\right\}$ is a sequence in $X$ such that $\lim _{n \rightarrow \infty} f x_{n}=\lim _{n \rightarrow \infty} g x_{n}=t$ for some $t \in X$. Thus, $f$ and $g$ are $f$-intimate mappings.

Similarly, we can show that $f$ and $g$ are $g$-intimate mappings.

Remark 2.4. A pair $f, g$ is $f$-intimate or $g$-intimate but, it does not hold compatible of type $(A)$, in general.

Example 2.5. Let $X=[0,1]$ with $d(x, y)=a^{|x-y|}$, where $x, y \in X, a>1$. and Define mappings $f$ and $g: X \rightarrow X$ by $f x=\frac{5}{x+5}$ and $g x=\frac{3}{x+3}$ for all $x \in X$.

Let $\left\{x_{n}\right\}=\left\{\frac{1}{n}\right\}$ be a sequence in $X$. Then $\lim _{n \rightarrow \infty} f x_{n}=\lim _{n \rightarrow \infty} g x_{n}=1$. Now

$$
\lim _{n \rightarrow \infty} d\left(f g x_{n}, f x_{n}\right)=a^{\frac{1}{6}} \quad \text { and } \quad \lim _{n \rightarrow \infty} d\left(g g x_{n}, g x_{n}\right)=a^{\frac{1}{4}} .
$$

Then

$$
\lim _{n \rightarrow \infty} d\left(f g x_{n}, f x_{n}\right) \leq \lim _{n \rightarrow \infty} d\left(g g x_{n}, g x_{n}\right)
$$

but

$$
\lim _{n \rightarrow \infty} d\left(f g x_{n}, g g x_{n}\right)=a^{\frac{1}{7}} \neq 1
$$

and

$$
\lim _{n \rightarrow \infty} d\left(g f x_{n}, f f x_{n}\right)=a^{\frac{1}{12}} \neq 1 .
$$

Hence $f$ and $g$ are $f$-intimate but not compatible of type $(A)$.

Proposition 2.6. Let $f$ and $g$ be mappings of a multiplicative metric space $(X, d)$ into itself. Assume that $f$ and $g$ are $g$-intimate and $f t=g t=p \in$ $X$. Then $d(g p, p) \leq d(f p, p)$. 
Proof. Suppose that $x_{n}=t$ for all $n \geq 1$. So $f x_{n}=g x_{n} \rightarrow f t=g t=p$. Since $f$ and $g$ are $g$-intimate, we have

$$
\begin{aligned}
d(g f t, g t) & =\lim _{n \rightarrow \infty} d\left(g f x_{n}, g x_{n}\right) \\
& \leq \lim _{n \rightarrow \infty} d\left(f f x_{n}, f x_{n}\right) \\
& =d(f f t, f t) .
\end{aligned}
$$

This implies that $d(g p, p) \leq d(f p, p)$.

In 2014, He et al. [4] proved the common fixed point theorem for pairs of weakly commuting mappings in a multiplicative metric space as follow:

Theorem 2.7. Let $A, B, S$ and $T$ be mappings of a complete multiplicative metric space $(X, d)$ into itself satisfying the following conditions:

$$
\begin{gathered}
S X \subset B X, \quad T X \subset A X ; \\
d(S x, T y) \leq[\max \{d(A x, B y), d(A x, S x), \\
\quad d(B y, T y), d(S x, B y), d(A x, T y)\}]^{\lambda}
\end{gathered}
$$

for all $x, y \in X$, where $\lambda \in\left(0, \frac{1}{2}\right)$;

(C3) one of the mappings $A, B, S$ and $T$ is continuous.

Assume that the pairs $A, S$ and $B, T$ are weakly commuting. Then $A, B, S$ and $T$ have a unique common fixed point.

In 2015, Kang et al. [5] prove common fixed point theorems for compatible mappings and its variants in a multiplicative metric space. Here we state the theorem only for compatible mappings of type $(A)$.

Theorem 2.8. Let $A, B, S$ and $T$ be mappings of a complete multiplicative metric space $(X, d)$ into itself satisfying the conditions $(C 1),(C 2)$ and $(C 3)$.

Assume that the pair $A, S$ and $B, T$ are compatible of type $(A)$. Then $A, B, S$ and $T$ have unique common fixed point.

Now we generalize Theorem 2.8 using intimate mappings in the following:

Theorem 2.9. Let $A, B, S$ and $T$ be mappings of a multiplicative metric space $(X, d)$ into itself satisfying the conditions $(C 1)$ and $(C 2)$.

Assume that $A(X)$ is complete and the pairs $A, S$ is $A$-intimate and $B, T$ is $B$-intimate. Then $A, B, S$ and $T$ have unique common fixed point. 
Proof. Let $x_{0} \in X$ be an arbitrary point. Since $S X \subset B X$, there exists $x_{1} \in X$ such that $S x_{0}=B x_{1}=y_{0}$. Now for this $x_{1}$ there exists $x_{2} \in X$ such that $T x_{1}=A x_{2}=y_{1}$. Similarly, we can inductively define a sequence $\left\{y_{n}\right\}$ such that

$$
y_{2 n}=S x_{2 n}=B x_{2 n+1}, \quad y_{2 n+1}=T x_{2 n+1}=A x_{2 n+2} .
$$

From the proof of [4, Theorem 3.1], $\left\{y_{n}\right\}$ is a multiplicative Cauchy sequence.

Now, suppose that $A X$ is complete there exists $p \in A X$ such that

$$
y_{2 n+1}=T x_{2 n+1}=A x_{2 n+2} \rightarrow p \quad \text { as } n \rightarrow \infty .
$$

Consequently, we can find $u \in X$ such that $A u=p$. Further a multiplicative Cauchy sequence $\left\{y_{n}\right\}$ has a convergent subsequence $\left\{y_{2 n+1}\right\}$, therefore, the sequence $\left\{y_{n}\right\}$ converges and hence a subsequence $\left\{y_{2 n}\right\}$ also converges. Thus we have

$$
y_{2 n}=S x_{2 n}=B x_{2 n+1} \rightarrow p \quad \text { as } n \rightarrow \infty .
$$

Now, we claim that $S u=p$. From inequality $\left(C_{2}\right)$ by putting $x=u$ and $y=x_{2 n+1}$ we have

$$
\begin{aligned}
& d\left(S u, T x_{2 n+1}\right) \\
& \leq\left[\operatorname { m a x } \left\{d\left(A u, B x_{2 n+1}\right), d(A u, S u),\right.\right. \\
& \left.\left.\quad d\left(B x_{2 n+1}, T x_{2 n+1}\right), d\left(S u, B x_{2 n+1}\right), d\left(A u, T x_{2 n+1}\right)\right\}\right]^{\lambda}
\end{aligned}
$$

Taking limit $n \rightarrow \infty$, we have

$$
\begin{aligned}
& d(S u, p) \\
& \leq[\max \{d(A u, p), d(A u, S u), d(p, p), d(S u, p), d(A u, p)\}]^{\lambda} \\
& =d^{\lambda}(S u, p) .
\end{aligned}
$$

This implies that $d(S u, p)=1$, that is, $S u=p$. So, $S u=A u=p$.

Next we claim that $p=T v$. Since $p=S u \in S X \subset B X$ there exists a point $v \in X$ such that $B v=p$. Putting $x=u$ and $y=v$ in inequality $\left(C_{2}\right)$ we have

$$
\begin{aligned}
d(p, T v)= & d(S u, T v) \\
\leq & {[\max \{d(A u, T v), d(A v, S u),} \\
& d(B v, T v), d(S u, B v), d(A u, T v)\}]^{\lambda} \\
= & {[\max \{d(p, T v), 1, d(p, T v), 1, d(p, T v)\}]^{\lambda} } \\
= & d^{\lambda}(p, T v) .
\end{aligned}
$$


This implies that $T v=B v=p$. Since $S u=A u=p$ and $A, S$ is $A$-intimate, by Proposition 2.6 we have $d(A p, p) \leq d(S p, p)$.

Suppose that $S p \neq p$. Then from inequality $\left(C_{2}\right)$, we get

$$
\begin{aligned}
& d(S p, p) \\
& =d(S p, T v) \\
& \leq[\max \{d(A p, B v), d(A p, S p), \\
& d(B v, T v), d(S p, B v), d(A p, T v)\}]^{\lambda} \\
& \leq[\max \{d(S p, p), d(A p, p) \cdot d(p, S p), 1, d(S p, p), d(S p, p)\}]^{\lambda} \\
& \leq[\max \{d(S p, p), d(S p, p) \cdot d(p, S p), 1, d(S p, p), d(S p, p)\}]^{\lambda} \\
& =d^{2 \lambda} d(S p, p) \text {. }
\end{aligned}
$$

This implies that $p=S p$ and also $A p=p$. Hence $A p=S p=p$.

Similarly, we get $B p=T p=p$.

The uniqueness follows easily from $(C 2)$. This completes the proof.

\section{References}

[1] M. Abbas, B. Ali, Y. I. Suleiman, Common fixed points of locally contractive mappings in multiplicative metric spaces with application, Int. J. Math. Math. Sci., 2015 (2015), Article ID 218683, 7 pages. doi:10.1155/2015/218683.

[2] A.E. Bashirov, E.M. Kurplnara, A. Ozyapici, Multiplicative calculus and its applications, J. Math. Anal. Appl., 337 (2008), 36-48. doi: 10.1016/j.jmaa.2007.03.081

[3] F. Gu, L.M. Cui, Y.H. Wu, Some fixed point theorems for new contractive type mappings, J. Qiqihar Univ., 19 (2013), 85-89.

[4] X. He, M. Song, D. Chen, Common fixed points for weak commutative mappings on a multiplicative metric space, Fixed Point Theory Appl., 48 (2014), 9 pages. doi: 10.1186/1687-1812-2014-48

[5] S.M. Kang, P. Kumar, S. Kumar, P. Nagpa, S.K. Garg, Common fixed points for compatible mappings and its variants in multiplicative metric spaces, Int. J. Pure Appl. Math., 102 (2015), 383-406. doi: 10.12732/ijpam.v102i2.14 
[6] M. Özavsar, A.C. Çevikel, Fixed points of multiplicative contraction mappings on multiplicative metric spaces, arXiv:1205.5131v1 [math.GM], 2012.

[7] D.R. Sahu, V.B. Dhagat, M. Srivastava, Fixed points with intimate mappings I, Bull. Calcutta Math. Soc., 93 (2001), 107-114.

[8] M. Sarwar, R. Badshah-e, Some unique fixed point theorems in multiplicative metric space, arXiv:1410.3384v2 [math.GM], 2014. 